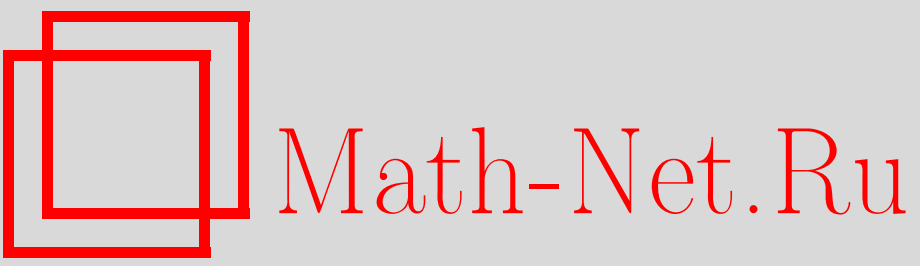

М. В. Шамолин, О предельных множествах дифференциальных уравнений около сингулярных особых точек, УМН, 2000, том 55, выпуск 3, 187-188

DOI: https://doi.org/10.4213/rm305

Использование Общероссийского математического портала Math-Net.Ru подразумевает, что вы прочитали и согласны с пользовательским соглашением

http://www.mathnet.ru/rus/agreement

Параметры загрузки:

IP: 3.80 .253 .173

26 апреля 2023 г., 14:41:47 


\title{
О ПРЕДЕЛЬНЫХ МНОЖЕСТВАХ ДИФФЕРЕНЦИАЛЬНЫХ УРАВНЕНИЙ ОКОЛО СИНГУЛЯРНЫХ ОСОБЫХ ТОЧЕК
}

\author{
М. В. ШАмолин
}

1. Классические методы нахождения замкнутых траекторий систем обыкновенных дифференциальных уравнений около (регулярных) особых точек восходят к работам А. Пуанкаре [1] (1892 г.). Позже исследования по данному вопросу были продолжены в работах А.А. Андронова [2], [3], Хопфа [4] и других авторов (например, известная бифуркация рождения цикла из слабого фокуса).

Отличительной особенностью работ отмеченных авторов является исследование окрестностей векторных полей систем именно около регулярной точки, т.е. там, где правые части систем имеют достаточное количество непрерывных производных.

Предположим, что у автономной системы третьего порядка в $\mathbb{R}^{3}\left\{x_{1}, x_{2}, x_{3}\right\}$ сушествует изолированная особая точка (начало координат). При этом на плоскости $\left\{\left(x_{1}, x_{2}, x_{3}\right) \in \mathbb{R}^{3}: x_{1}=0\right\}$ правая часть системы, вообще говоря, не определена (например, в правой части присутствует особенность типа $f\left(x_{1}, x_{2}, x_{3}\right) \frac{1}{x_{1}}$, где $f\left(x_{1}, x_{2}, x_{3}\right)$ - достаточно гладкая функция около начала координат).

Предположим, что плоскость $\left\{\left(x_{1}, x_{2}, x_{3}\right) \in \mathbb{R}^{3}: x_{3}=0\right\}$ является интегральной для независимой подсистемы

$$
x_{1}^{\prime}=\Phi_{1}\left(x_{1}, x_{2}\right), \quad x_{2}^{\prime}=\Phi_{2}\left(x_{1}, x_{2}\right)
$$

общей системы третьего порядка после формального доопределения последней на всей плоскости $\left\{\left(x_{1}, x_{2}, x_{3}\right) \in \mathbb{R}^{3}: x_{3}=0\right\}$.

2. В приложениях могут возникать случаи, когда правая часть системы возле особой точки или не имеет производных, или их нахождение сильно затруднительно, поскольку возникает проблема "доопределения по непрерывности" правых частей системы в самих особых точках.

Если на плоскости $\left\{\left(x_{1}, x_{2}, x_{3}\right) \in \mathbb{R}^{3}: x_{3}=0\right\}$ у системы (1) вблизи начала координат есть предельный цикл, то возникает вопрос: появятся ли у общей системы третьего порядка в области $\left\{\left(x_{1}, x_{2}, x_{3}\right) \in \mathbb{R}^{3}: x_{1}>0, x_{3}>0\right\}$ какие-либо нетривиальные предельные множества? В общем случае данный вопрос достаточно сложный, но, используя трехмерную топографическую систему Пуанкаре [5] как совокупность (двумерных) поверхностей уровня функции $V=x_{1}^{2 k}+x_{2}^{2 k}+x_{3}^{2 k}, k \in \mathbb{N}$, вблизи начала координат и исследуя знак скалярного произведения [5] (grad $V(x), \nu)$, где $\nu$ - векторное поле исследуемой трехмерной системы, можно "поймать" предельные циклы не только вблизи особой точки.

Рассмотрим для начала пример системы третьего порядка, возникающий в динамике твердого тела, взаимодействующего со средой. Систему

$$
\begin{aligned}
& \alpha^{\prime}=-Z_{2}+\sigma\left(Z_{1}^{2}+Z_{2}^{2}\right) \sin \alpha+\sigma n_{0}^{2} \sin \alpha \cos ^{2} \alpha+\frac{B}{m} \sin \alpha \cos \alpha, \\
& Z_{2}^{\prime}=n_{0}^{2} \sin \alpha \cos \alpha-Z_{2} \Psi\left(\alpha, Z_{1}, Z_{2}\right)-Z_{1}^{2} \frac{\cos \alpha}{\sin \alpha}, \\
& Z_{1}^{\prime}=-Z_{1} \Psi\left(\alpha, Z_{1}, Z_{2}\right)+Z_{1} Z_{2} \frac{\cos \alpha}{\sin \alpha},
\end{aligned}
$$

где $\Psi\left(\alpha, Z_{1}, Z_{2}\right)=-\sigma\left(Z_{1}^{2}+Z_{2}^{2}\right) \cos \alpha+\sigma n_{0}^{2} \sin ^{2} \alpha \cos \alpha-\frac{B}{m} \cos ^{2} \alpha$, рассмотрим в слое $\Pi_{(0, \pi)}=$ $\left\{\left(\alpha, Z_{1}, Z_{2}\right) \in \mathbb{R}^{3}: Z_{1}>0,0<\alpha<\pi\right\}, \sigma, n_{0}, B, m>0$.

Если формально положить $Z_{1}=0$, а систему (2) доопределить по непрерывности при $Z_{1}=0$, то плоскость $\left\{\left(\alpha, Z_{1}, Z_{2}\right) \in \mathbb{R}^{3}: Z_{1}=0\right\}$ является интегральной. На ней "отшепившаяся" система второго порядка

$$
\begin{aligned}
& \alpha^{\prime}=-\Omega+\sigma \Omega^{2} \sin \alpha+\sigma n_{0}^{2} \sin \alpha \cos ^{2} \alpha+\frac{B}{m} \sin \alpha \cos \alpha \\
& \Omega^{\prime}=n_{0}^{2} \sin \alpha \cos \alpha+\Omega\left\{\sigma \Omega^{2} \cos \alpha-\sigma n_{0}^{2} \sin ^{2} \alpha \cos \alpha+\frac{B}{m} \cos ^{2} \alpha\right\}
\end{aligned}
$$


на двумерном цилиндре $\left\{(\alpha, \Omega) \in \mathbb{R}^{2}: \alpha \bmod 2 \pi\right\}$ имеет бесконечное число топологически различных фазовых портретов [6], [7], и при некоторых условиях вокруг точки $(\pi, 0)$ могут возникнуть предельные циклы [7].

Возникает вопрос: появятся ли у системы $(2)$ в замыкании слоя $\overline{\Pi_{(0, \pi)}}$ около особой точки $(\pi, 0,0)$ какие-либо замкнутые траектории или, вообще говоря, предельные множества? Воспользуемся методом многомерных топографических систем (Пуанкаре) [5]. Рассмотрим поверхность уровня (топографиическую систему) неотрицательной функции (Ляпунова)

$$
V_{0}\left(\alpha, Z_{1}, Z_{2}\right)=Z_{1}^{2}+Z_{2}^{2}+n_{0}^{2} \sin ^{2} \alpha
$$

во всем пространстве $\mathbb{R}^{3}\left(\alpha, Z_{1}, Z_{2}\right)$. Поверхности уровня функции $V_{0}$ являются интегральными уровнями для системы

$$
\alpha^{\prime}=-Z_{2}, \quad Z_{2}^{\prime}=n_{0}^{2} \sin \alpha \cos \alpha-Z_{1}^{2} \frac{\cos \alpha}{\sin \alpha}, \quad Z_{1}^{\prime}=Z_{1} Z_{2} \frac{\cos \alpha}{\sin \alpha},
$$

которую рассматриваем в качестве системы сравнения для (2). Скалярное произведение градиента $\operatorname{grad} V_{0}\left(\alpha, Z_{1}, Z_{2}\right)$ на векторное поле системы $(2)$ в координатах $\left(\alpha, Z_{1}, Z_{2}\right)$ является знакопеременной характеристической функцией $\chi\left(\alpha, Z_{1}, Z_{2}\right)$ при $2 \frac{B}{m n_{0}}=\sigma n_{0}+\varepsilon$, где $\varepsilon$ - малое положительное число. В некоторой малой окрестности точки $(\pi, 0,0)$ характеристическая функция [5] $\chi$ положительно определена, что и подтверждает отталкивающий характер (сингулярной) особой точки $(\pi, 0,0)$. При этом существует сферический слой в малой окрестности рассматриваемой особой точки, в которой через его внутреннюю и внешнюю границы фазовые траектории системы $(2)$ только входят. Поскольку вблизи $(\pi, 0,0)$ других особых точек системы $(2)$ нет, в рассматриваемом сферическом слое существует нетривиальное $\omega$-предельное множество. Можно показать, что в данном случае это предельное множество - притягивающий предельный цикл.

3. Приведенные рассуждения легко распространяются и на более общий случай маятниковых систем вида

$$
\begin{aligned}
\varphi^{\prime} & =-x_{1}+\delta F_{\varphi}\left(\varphi, x_{1}, x_{2}\right), \\
x_{1}^{\prime} & =F(\varphi)-x_{2}^{2} \operatorname{ctg} \varphi+\delta F_{1}\left(\varphi, x_{1}, x_{2}, \operatorname{ctg} \varphi\right), \\
x_{2}^{\prime} & =x_{1} x_{2} \operatorname{ctg} \varphi+\delta F_{2}\left(\varphi, x_{1}, x_{2}, \operatorname{ctg} \varphi\right),
\end{aligned}
$$

где $F^{\prime}(0)>0, F_{i}\left(s_{1}, s_{2}, s_{3}, s_{4}\right)$ - гладкие функции (вблизи начала координат), $\frac{\partial F_{i}}{\partial s_{4}}\left(s_{1}, s_{2}, s_{3}, s_{4}\right)$ - полиномы по $s_{4}$ степени $s^{i}, i=1,2, \delta \in \mathbb{R}$. При этом функцию $V$ следует искать в виде

$$
V=f_{\varphi}\left(\varphi, x_{1}, x_{2}\right) \sin ^{2} \varphi+f_{1}\left(\varphi, x_{1}, x_{2}\right) x_{1}^{2 k}+f_{2}\left(\varphi, x_{1}, x_{2}\right) x_{2}^{2 k},
$$

$f_{\varphi}, f_{1}, f_{2}$ - гладкие положительные функции и $2 k \geqslant s+1, s=\max _{i=1,2} s^{i}$.

\section{СПИСОК ЛИТЕРАТУРЫ}

[1] Пуанкаре А. О кривых, определяемых дифференциальными уравнениями. М.-Л.: ОГИЗ, 1947. [2] Андронов А. А. Собрание трудов. М.: Изд-во АН СССР, 1956. [3] Андронов А.А., Леонтович Е.А. Некоторые случаи зависимости предельных циклов от параметра // Ученые записки ГГУ. 1937. №6. [4] Hopf E. Abzweigung einer periodischen Losung von einer stationaren Losung eines Differential systems // Ber. Math. - Phys. Sachsische Academie der Wissenschaften Leipzig. 1942. V. 94. Р. 1-22. [5] Шамолин М. В. // УМН. 1997. Т. 52. № 3. С. 177-178. [6] Шамолин М. В. // Докл. РАН. 1994. Т. 337. № 5. С. 611-614. [7] Шамолин М. В. // Докл. РАН. 1996. Т. 349. № 2. С. 193-197. 